\title{
Complex Wave Solutions to Mathematical Biology Models II: Two Dimensional Fisher and Nagumo Equations
}

\author{
Alper Korkmaz* \\ Çankırı Karatekin University, Department of Mathematics, 18200, Çankırı, Turkey.
}

\begin{abstract}
We extended the usage of the expansion method based on Sine-Gordon equation to the two dimensional Fisher equation. The relation between the trigonometric and hyperbolic functions are derived from the Sine-Gordon equation defined in two space dimension. The complex-valued traveling wave solutions to the two dimensional Fisher and Nagumo equations are set in forms a finite series of multiplications of powers of $\operatorname{sech}($.$) and \tanh ($. functions.
\end{abstract}

Keywords: Reaction-Diffusion Equations; Two Dimensional Fisher Equation; Nagumo Equation; Kolmogorov-Petrovsky-Piskunov Equation; Traveling Wave Solution. MSC2010: 35C07;35R11;35Q53.

PACS: 02.30.Jr; 02.70.Wz; 04.20.Jb

\section{Introduction}

Since Fisher [1] defined the deterministic form of a stochastic model [2] in one dimension as

$$
v_{t}+p v_{x_{1} x_{1}}+q v(v-s)=0
$$

where real $p$ denotes linear diffusion coefficient and $q$ is the rate of reproduction, various forms of reaction-diffusion equations have been described to explain different biological, physical or chemical phenomena. Fisher's equation (FE) defined

*alperkorkmaz7@gmail.com 
in (1) describes spatial spread of an advantageous gene in a population living in a linear one dimensional habitat [1-3]. A more general form of the FE (2) was studied by Kolmogorov et al. at the same year [4]. A two-dimensional form of the same model is given in the form

$$
u_{t}+p u_{x_{1} x_{1}}+q u_{x_{2} x_{2}}+r u(u-s)=0
$$

where $p, q$ diffusion coefficients in $x_{1}$ and $x_{2}$ directions, respectively, $r$ and $s$ are non zero parameters. Independent on its space dimension, a general form of the equation is also known as Kolmogorov-Petrovsky-Piskunov (KPP) or Fisher-KPP equation. In the two dimensional form of the $\mathrm{FE}$, we have relaxed the coefficients $p$ and $q$ denoting diffusion in $x_{1}$ and $x_{2}$ dimensions, respectively, instead of defining the diffusion term $\Delta u$ where $\Delta$ represents Laplace operator.

General solution for a particular wave speed was determined in the case having Painlevé type solutions [5]. Some exact solutions to the FE were constructed in terms of powers of hyperbolic tangent functions [6]. Wave type solutions were found by using exp-function approach in [7]. Some more exact solutions in complex forms were suggested by the first integral method [8]. Kudryashov method was also implemented to the FE to generate some traveling wave type exact solutions in some rational function forms of exponential function series in both numerator and denominator [9].

The Nagumo Equation (NE) of the form

$$
u_{t}+p u_{x_{1} x_{1}}+q u(1-u)(u-r)=0
$$

where $p$ is the coefficient of diffusion, $q$ and $r$ real non-zero parameters [10,11]. The constant solutions $u=1$ and $u=r$ to the NE (3) are stable and $u=0$ is unstable for $-1 \leq r<0$. On the other hand, $u=0$ and $u=1$ both are stable while $u=r$ is unstable in $r \in[0,1)$ [11]. Various techniques are implemented to the NE (3) to set the solutions. Exp-function [12,13], Cole-Hopf transformation [14], direct method [15], simple equation approach [16], the method of first integral [17] are well known approaches to derive traveling single or multi wave solutions. Since the aim of this study does not examine the stability of constant solutions, $r$ is relaxed by assuming only a non-zero constant.

Different from classical approaches in literature, an expansion technique based on Sine-Gordon equation is constructed to find the traveling wave solutions to the 2D-Fisher's equation and the Nagumo equation. Before deriving the solutions, some preliminaries are given below. 


\section{Expansion Method based on Sine-Gordon Equa- tion}

Consider a general form of the Sine-Gordon equation in $N$ dimension:

$$
\Delta u-u_{t t}=m^{2} \sin u, \quad m \text { is constant }
$$

where $u=u\left(x_{1}, x_{2}, \ldots, x_{N}, t\right)$. The traveling wave transform

$$
\begin{gathered}
u\left(x_{1}, x_{2}, \ldots, x_{N} \ldots, t\right) \rightarrow U(\xi) \\
\xi=a\left(x_{1}+x_{2}+\ldots+x_{N}-\nu t\right)
\end{gathered}
$$

reduces the $\mathrm{SGE}(4)$ to

$$
\frac{d^{2} U}{d \xi^{2}}=\frac{m^{2}}{a^{2}\left(N-\nu^{2}\right)} \sin U
$$

where $\nu$ is the parameter of velocity of the traveling wave defined in the compatible traveling wave transform [18]. Some calculations reduces the previous equation to

$$
\left(\frac{d(U / 2)}{d \xi}\right)^{2}=\frac{m^{2}}{a^{2}\left(N-\nu^{2}\right)} \sin ^{2} U / 2+\tilde{C}
$$

where $\tilde{C}$ is constant of integration that is assumed zero in the study. The assumptions $w(\xi)=U(\xi) / 2$ and unit $m^{2} /\left(a^{2}\left(N-\nu^{2}\right)\right)$ leads the conversion of $(7)$ to the form

$$
\frac{d(w)}{d \xi}=\sin w
$$

Thus, (8) gives the following relations

$$
\sin w(\xi)=\left.\frac{2 c e^{\xi}}{c^{2} e^{2 \xi}+1}\right|_{c=1}=\operatorname{sech} \xi
$$

or

$$
\cos w(\xi)=\left.\frac{c^{2} e^{2 \xi}-1}{c^{2} e^{2 \xi}+1}\right|_{c=1}=-\tanh \xi
$$

where $c \neq 0$ is integral constant.

\section{The initial step}

The compatible wave transform explained above reduces the governing PDE

$$
\Omega\left(u, u_{x_{1}}, u_{x_{2}}, \ldots, u_{t}, u_{x_{1} x_{2}}, \ldots\right)=0
$$


to an ODE

$$
\tilde{\Omega}\left(U, U^{\prime}, U^{\prime \prime}, \ldots\right)=0
$$

with the transform variable $\xi=a\left(x_{1}+x_{2}+\ldots+x_{N}-\nu t\right)$. Suppose that

$$
U(\xi)=A_{0}+\sum_{i=1}^{Q} \tanh ^{i-1}(\xi)\left(B_{i} \operatorname{sech} \xi+A_{i} \tanh \xi\right)
$$

solves (12). Then, the relations (9)-(10) changes the form of the solution in terms of $w$ as

$$
U(w)=A_{0}+\sum_{i=1}^{Q} \cos ^{i-1}(w)\left(B_{i} \sin w+A_{i} \cos w\right)
$$

The determination of upper index $Q$ requires the balancing procedure between the highest ordered nonlinear and the highest ordered derivative terms. Determining $Q$ gives the degree of the finite power series solution in the form of multiplication of $\sin$ and cos functions. The predicted solution (14) is substituted into (12), the coefficients of powers of trigonometric functions are equated to zero. The resultant system of algebraic equations are solved for non zero $a$ and $\nu$ with at least one of $A_{Q}$ or $B_{Q}$ is non zero. The next step is to set the solutions $U(w)$ by using the relations between the determined parameters. Returning back to $\xi$ changes the solutions to hyperbolic functions. In the final step, the solutions are expressed in the original variables $x_{i}, 1 \leq i \leq N$ and $t$.

\section{Solutions to the Fisher Equation in two dimen- sion}

The traveling wave transform

$$
u\left(x_{1}, x_{2}, t\right) \rightarrow U(\xi), \xi=a\left(x_{1}+x_{2}-\nu t\right)
$$

reduces the two dimensional FE (2) to

$$
-a \nu U^{\prime}+(p+q) a^{2} U^{\prime \prime}+r U(U-s)=0
$$

where prime notation denotes the derivative order in $\xi$. The balancing procedure between $U^{\prime \prime}$ and $U^{2}$ gives $Q=2$. Thus, the predicted solution should be of the form

$$
U(w)=A_{0}+A_{1} \cos w+B_{1} \sin w+A_{2} \cos ^{2} w+B_{2} \cos w \sin w
$$


with $A_{2}^{2}+B_{2}^{2} \neq 0$. Substitution of the predicted solution (17) into (16) gives

$$
\begin{aligned}
& r A_{2}^{2}(\cos (w(\xi)))^{4}+\left((p+q) a^{2} B_{2}+2 r B_{2} A_{2}\right)(\cos (w(\xi)))^{3} \sin (w(\xi)) \\
& +2 r A_{1} A_{2}(\cos (w(\xi)))^{3}+\left(-4(p+q) a^{2} A_{2}+r B_{2}^{2}\right)(\sin (w(\xi)))^{2}(\cos (w(\xi)))^{2} \\
& +\left(-a \nu B_{2}+(p+q) a^{2} B_{1}+2 r B_{1} A_{2}+2 r A_{1} B_{2}\right)(\cos (w(\xi)))^{2} \sin (w(\xi)) \\
& +\left(r A_{0} A_{2}+r A_{1}^{2}+r A_{2}\left(-s+A_{0}\right)\right)(\cos (w(\xi)))^{2}-5(p+q) a^{2} B_{2} \cos (w(\xi))(\sin (w(\xi)))^{3} \\
& +\left(2 a \nu A_{2}-2(p+q) a^{2} A_{1}+2 r B_{1} B_{2}\right) \cos (w(\xi))(\sin (w(\xi)))^{2} \\
& +\left(-a \nu B_{1}+r A_{0} B_{2}+2 r B_{1} A_{1}+r B_{2}\left(-s+A_{0}\right)\right) \cos (w(\xi)) \sin (w(\xi)) \\
& +\left(r A_{0} A_{1}+r A_{1}\left(-s+A_{0}\right)\right) \cos (w(\xi))+2(p+q) a^{2} A_{2}(\sin (w(\xi)))^{4} \\
& +\left(a \nu B_{2}-(p+q) a^{2} B_{1}\right)(\sin (w(\xi)))^{3}+\left(a \nu A_{1}+r B_{1}^{2}\right)(\sin (w(\xi)))^{2} \\
& +\left(r A_{0} B_{1}+r B_{1}\left(-s+A_{0}\right)\right) \sin (w(\xi))+r A_{0}\left(-s+A_{0}\right)=0
\end{aligned}
$$

Using the relation between $\cos ($.$) and \sin ($.$) functions the number of coefficients$ is easily reduced. Equating each coefficient of powers of multiplication $\cos ($.$) and$ $\sin ($.$) functions in the resultant equation, the following system of equations are$ constructed:

$$
\begin{aligned}
2 a^{2} A_{2} p+2 a^{2} A_{2} q+a \nu A_{1}-r A_{0} s+r A_{0}{ }^{2}+r B_{1}{ }^{2} & =0 \\
-a^{2} p B_{1}-a^{2} q B_{1}+a \nu B_{2}-r s B_{1}+2 r A_{0} B_{1} & =0 \\
6 a^{2} p B_{2}+6 a^{2} q B_{2}+2 r A_{2} B_{2} & =0 \\
2 a^{2} p B_{1}+2 a^{2} q B_{1}-2 a \nu B_{2}+2 r A_{1} B_{2}+2 r A_{2} B_{1} & =0 \\
-5 a^{2} p B_{2}-5 a^{2} q B_{2}-a \nu B_{1}-r s B_{2}+2 r A_{0} B_{2}+2 r A_{1} B_{1} & =0 \\
-8 a^{2} A_{2} p-8 a^{2} A_{2} q-a \nu A_{1}-r A_{2} s+2 r A_{0} A_{2}+r{A_{1}}^{2}-r B_{1}{ }^{2}+r B_{2}{ }^{2} & =0 \\
6 a^{2} A_{2} p+6 a^{2} A_{2} q+r A_{2}{ }^{2}-r B_{2}{ }^{2} & =0 \\
2 a^{2} A_{1} p+2 a^{2} A_{1} q-2 a \nu A_{2}+2 r A_{1} A_{2}-2 r B_{1} B_{2} & =0 \\
-2 a^{2} A_{1} p-2 a^{2} A_{1} q+2 a \nu A_{2}-r A_{1} s+2 r A_{0} A_{1}+2 r B_{1} B_{2} & =0
\end{aligned}
$$

Solving the system given above for $a, \nu, A_{0}, A_{1}, A_{2}, B_{1}$ and $B_{2}$ by considering $A_{2}^{2}+B_{2}^{2} \neq 0$ gives the solutions satisfying required conditions on parameters expressed above. The acceptable solutions are tabulated in Table 1 . Using the relations between the parameters given in Table 1, the solutions of two dimensional 
Table 1: The solution of the algebraic system of equations derived for two dimensional Fisher equation

\begin{tabular}{ccccccc}
\hline \hline$a$ & $\nu$ & $A_{0}$ & $A_{1}$ & $A_{2}$ & $B_{1}$ & $B_{2}$ \\
\hline$\frac{\sqrt{6 r s}}{6 \sqrt{p+q}}$ & $\frac{5 \sqrt{6 r s(p+q)}}{6}$ & $s$ & $\frac{s}{2}$ & $-\frac{s}{2}$ & $-\frac{s}{2} i$ & $\frac{s}{2} i$ \\
$-\frac{\sqrt{6 r s}}{6 \sqrt{p+q}}$ & $-\frac{5 \sqrt{6 r s(p+q)}}{6}$ & $s$ & $\frac{s}{2}$ & $-\frac{s}{2}$ & $-\frac{s}{2} i$ & $\frac{s}{2} i$ \\
$\frac{\sqrt{6 r s}}{6 \sqrt{p+q}}$ & $\frac{5 \sqrt{6 r s(p+q)}}{6}$ & $s$ & $\frac{s}{2}$ & $-\frac{s}{2}$ & $\frac{s}{2} i$ & $-\frac{s}{2} i$ \\
$-\frac{\sqrt{6 r s}}{6 \sqrt{p+q}}$ & $-\frac{5 \sqrt{6 r s(p+q)}}{6}$ & $s$ & $\frac{s}{2}$ & $-\frac{s}{2}$ & $\frac{s}{2} i$ & $-\frac{s}{2} i$ \\
$\frac{\sqrt{6 r s}}{6 \sqrt{p+q}}$ & $-\frac{5 \sqrt{6 r s(p+q)}}{6}$ & $s$ & $-\frac{s}{2}$ & $-\frac{s}{2}$ & $\frac{s}{2} i$ & $\frac{s}{2} i$ \\
$-\frac{\sqrt{6 r s}}{6 \sqrt{p+q}}$ & $\frac{5 \sqrt{6 r s(p+q)}}{6}$ & $s$ & $-\frac{s}{2}$ & $-\frac{s}{2}$ & $\frac{s}{2} i$ & $\frac{s}{2} i$ \\
$\frac{\sqrt{6 r s}}{6 \sqrt{p+q}}$ & $-\frac{5 \sqrt{6 r s(p+q)}}{6}$ & $s$ & $-\frac{s}{2}$ & $-\frac{s}{2}$ & $-\frac{s}{2} i$ & $-\frac{s}{2} i$ \\
$-\frac{\sqrt{6 r s}}{6 \sqrt{p+q}}$ & $\frac{5 \sqrt{6 r s(p+q)}}{6}$ & $s$ & $-\frac{s}{2}$ & $-\frac{s}{2}$ & $-\frac{s}{2} i$ & $-\frac{s}{2} i$ \\
$\frac{\sqrt{6 r s}}{6 \sqrt{p+q}} i$ & $\frac{5 \sqrt{6 r s(p+q)}}{6} i$ & 0 & $-\frac{s}{2}$ & $\frac{s}{2}$ & $-\frac{s}{2} i$ & $\frac{s}{2} i$ \\
$-\frac{\sqrt{6 r s}}{6 \sqrt{p+q}} i$ & $-\frac{5 \sqrt{6 r s(p+q)}}{6} i$ & 0 & $-\frac{s}{2}$ & $\frac{s}{2}$ & $-\frac{s}{2} i$ & $\frac{s}{2} i$ \\
$\frac{\sqrt{6 r s}}{6 \sqrt{p+q}} i$ & $\frac{5 \sqrt{6 r s(p+q)}}{6} i$ & 0 & $-\frac{s}{2}$ & $\frac{s}{2}$ & $-\frac{s}{2} i$ & $-\frac{s}{2} i$ \\
$-\frac{\sqrt{6 r s}}{6 \sqrt{p+q}} i$ & $-\frac{5 \sqrt{6 r s(p+q)}}{6} i$ & 0 & $-\frac{s}{2}$ & $\frac{s}{2}$ & $-\frac{s}{2} i$ & $-\frac{s}{2} i$ \\
$\frac{\sqrt{6 r s}}{6 \sqrt{p+q}} i$ & $-\frac{5 \sqrt{6 r s(p+q)}}{6} i$ & 0 & $\frac{s}{2}$ & $\frac{s}{2}$ & $\frac{s}{2} i$ & $\frac{s}{2} i$ \\
$-\frac{\sqrt{6 r s}}{6 \sqrt{p+q}} i$ & $\frac{5 \sqrt{6 r s(p+q)}}{6} i$ & 0 & $\frac{s}{2}$ & $\frac{s}{2}$ & $\frac{s}{2} i$ & $\frac{s}{2} i$ \\
$\frac{\sqrt{6 r s}}{6 \sqrt{p+q}} i$ & $-\frac{5 \sqrt{6 r s(p+q)}}{6} i$ & 0 & $\frac{s}{2}$ & $\frac{s}{2}$ & $-\frac{s}{2} i$ & $-\frac{s}{2} i$ \\
$-\frac{\sqrt{6 r s}}{6 \sqrt{p+q}} i$ & $\frac{5 \sqrt{6 r s(p+q)}}{6} i$ & 0 & $\frac{s}{2}$ & $\frac{s}{2}$ & $-\frac{s}{2} i$ & $-\frac{s}{2} i$ \\
$\frac{\sqrt{6 r s}}{12 \sqrt{p+q}} i$ & $\frac{5 \sqrt{6 r s(p+q)}}{6} i$ & $\frac{s}{4}$ & $-\frac{s}{2}$ & $\frac{s}{4}$ & 0 & 0 \\
$-\frac{\sqrt{6 r s}}{12 \sqrt{p+q}} i$ & $-\frac{5 \sqrt{6 r s(p+q)}}{6} i$ & $\frac{s}{4}$ & $-\frac{s}{2}$ & $\frac{s}{4}$ & 0 & 0 \\
$\frac{\sqrt{6 r s}}{12 \sqrt{p+q}} i$ & $-\frac{5 \sqrt{6 r s(p+q)}}{6} i$ & $\frac{s}{4}$ & $\frac{s}{2}$ & $\frac{s}{4}$ & 0 & 0 \\
$-\frac{\sqrt{6 r s}}{12 \sqrt{p+q}} i$ & $\frac{5 \sqrt{6 r s(p+q)}}{6} i$ & $\frac{s}{4}$ & $-\frac{s}{2}$ & $\frac{s}{4}$ & 0 & 0 \\
$\frac{\sqrt{6 r s}}{12 \sqrt{p+q}} i$ & $-\frac{5 \sqrt{6 r s(p+q)}}{6} i$ & $\frac{3 s}{4}$ & $-\frac{s}{2}$ & $-\frac{s}{4}$ & 0 & 0 \\
$-\frac{\sqrt{6 r s}}{12 \sqrt{p+q}}$ & $\frac{5 \sqrt{6 r s(p+q)}}{6}$ & $\frac{3 s}{4}$ & $-\frac{s}{2}$ & $-\frac{s}{4}$ & 0 & 0 \\
$\frac{\sqrt{6 r s}}{12 \sqrt{p+q}}$ & $\frac{5 \sqrt{6 r s(p+q)}}{6}$ & $\frac{3 s}{4}$ & $\frac{s}{2}$ & $-\frac{s}{4}$ & 0 & 0 \\
\hline \hline
\end{tabular}


Fisher equation (2) are written in explicit forms:

$$
\begin{aligned}
& u_{1}\left(x_{1}, x_{2}, t\right)=s-\frac{s}{2} \tanh \kappa-\frac{s}{2} i \operatorname{sech} \kappa-\frac{s}{2} \tanh ^{2} \kappa-\frac{s}{2} i \tanh \kappa \operatorname{sech} \kappa, \kappa=\frac{\sqrt{6 r s}}{6 \sqrt{p+q}}\left(x_{1}+x_{2}-\frac{5 \sqrt{6 r s(p+q)}}{6}\right) t \\
& u_{2}\left(x_{1}, x_{2}, t\right)=s-\frac{s}{2} \tanh \kappa-\frac{s}{2} i \operatorname{sech} \kappa-\frac{s}{2} \tanh ^{2} \kappa-\frac{s}{2} i \tanh \kappa \operatorname{sech} \kappa, \kappa=-\frac{\sqrt{6 r s}}{6 \sqrt{p+q}}\left(x_{1}+x_{2}+\frac{5 \sqrt{6 r s(p+q)}}{6}\right) t \\
& u_{3}\left(x_{1}, x_{2}, t\right)=s-\frac{s}{2} \tanh \kappa+\frac{s}{2} i \operatorname{sech} \kappa-\frac{s}{2} \tanh ^{2} \kappa+\frac{s}{2} i \tanh \kappa \operatorname{sech} \kappa, \kappa=\frac{\sqrt{6 r s}}{6 \sqrt{p+q}}\left(x_{1}+x_{2}-\frac{5 \sqrt{6 r s(p+q)}}{6}\right) t \\
& u_{4}\left(x_{1}, x_{2}, t\right)=s-\frac{s}{2} \tanh \kappa+\frac{s}{2} i \operatorname{sech} \kappa-\frac{s}{2} \tanh ^{2} \kappa+\frac{s}{2} i \tanh \kappa \operatorname{sech} \kappa, \kappa=-\frac{\sqrt{6 r s}}{6 \sqrt{p+q}}\left(x_{1}+x_{2}+\frac{5 \sqrt{6 r s(p+q)}}{6}\right) t \\
& u_{5}\left(x_{1}, x_{2}, t\right)=s+\frac{s}{2} \tanh \kappa+\frac{s}{2} i \operatorname{sech} \kappa-\frac{s}{2} \tanh ^{2} \kappa-\frac{s}{2} i \tanh \kappa \operatorname{sech} \kappa, \kappa=\frac{\sqrt{6 r s}}{6 \sqrt{p+q}}\left(x_{1}+x_{2}+\frac{5 \sqrt{6 r s(p+q)}}{6}\right) t \\
& u_{6}\left(x_{1}, x_{2}, t\right)=s+\frac{s}{2} \tanh \kappa+\frac{s}{2} i \operatorname{sech} \kappa-\frac{s}{2} \tanh ^{2} \kappa-\frac{s}{2} i \tanh \kappa \operatorname{sech} \kappa, \kappa=-\frac{\sqrt{6 r s}}{6 \sqrt{p+q}}\left(x_{1}+x_{2}-\frac{5 \sqrt{6 r s(p+q)}}{6}\right) t \\
& u_{7}\left(x_{1}, x_{2}, t\right)=s+\frac{s}{2} \tanh \kappa-\frac{s}{2} i \operatorname{sech} \kappa-\frac{s}{2} \tanh ^{2} \kappa+\frac{s}{2} i \tanh \kappa \operatorname{sech} \kappa, \kappa=\frac{\sqrt{6 r s}}{6 \sqrt{p+q}}\left(x_{1}+x_{2}+\frac{5 \sqrt{6 r s(p+q)}}{6}\right) t \\
& u_{8}\left(x_{1}, x_{2}, t\right)=s+\frac{s}{2} \tanh \kappa-\frac{s}{2} i \operatorname{sech} \kappa-\frac{s}{2} \tanh ^{2} \kappa+\frac{s}{2} i \tanh \kappa \operatorname{sech} \kappa, \kappa=-\frac{\sqrt{6 r s}}{6 \sqrt{p+q}}\left(x_{1}+x_{2}-\frac{5 \sqrt{6 r s(p+q)}}{6}\right) t \\
& u_{9}\left(x_{1}, x_{2}, t\right)=\frac{s}{2} \tanh \kappa-\frac{s}{2} i \operatorname{sech} \kappa+\frac{s}{2} \tanh ^{2} \kappa-\frac{s}{2} i \tanh \kappa \operatorname{sech} \kappa, \kappa=\frac{\sqrt{6 r s}}{6 \sqrt{p+q}} i\left(x_{1}+x_{2}-\frac{5 \sqrt{6 r s(p+q)}}{6}\right) i t \\
& u_{10}\left(x_{1}, x_{2}, t\right)=\frac{s}{2} \tanh \kappa-\frac{s}{2} i \operatorname{sech} \kappa+\frac{s}{2} \tanh ^{2} \kappa-\frac{s}{2} i \tanh \kappa \operatorname{sech} \kappa, \kappa=-\frac{\sqrt{6 r s}}{6 \sqrt{p+q}} i\left(x_{1}+x_{2}+\frac{5 \sqrt{6 r s(p+q)}}{6}\right) i t \\
& u_{11}\left(x_{1}, x_{2}, t\right)=\frac{s}{2} \tanh \kappa-\frac{s}{2} i \operatorname{sech} \kappa+\frac{s}{2} \tanh ^{2} \kappa+\frac{s}{2} i \tanh \kappa \operatorname{sech} \kappa, \kappa=\frac{\sqrt{6 r s}}{6 \sqrt{p+q}} i\left(x_{1}+x_{2}-\frac{5 \sqrt{6 r s(p+q)}}{6}\right) i t \\
& u_{12}\left(x_{1}, x_{2}, t\right)=\frac{s}{2} \tanh \kappa-\frac{s}{2} i \operatorname{sech} \kappa+\frac{s}{2} \tanh ^{2} \kappa+\frac{s}{2} i \tanh \kappa \operatorname{sech} \kappa, \kappa=-\frac{\sqrt{6 r s}}{6 \sqrt{p+q}} i\left(x_{1}+x_{2}+\frac{5 \sqrt{6 r s(p+q)}}{6}\right) i t \\
& u_{13}\left(x_{1}, x_{2}, t\right)=-\frac{s}{2} \tanh \kappa+\frac{s}{2} i \operatorname{sech} \kappa+\frac{s}{2} \tanh ^{2} \kappa-\frac{s}{2} i \tanh \kappa \operatorname{sech} \kappa, \kappa=\frac{\sqrt{6 r s}}{6 \sqrt{p+q}} i\left(x_{1}+x_{2}+\frac{5 \sqrt{6 r s(p+q)}}{6}\right) i t \\
& u_{14}\left(x_{1}, x_{2}, t\right)=-\frac{s}{2} \tanh \kappa+\frac{s}{2} i \operatorname{sech} \kappa+\frac{s}{2} \tanh ^{2} \kappa-\frac{s}{2} i \tanh \kappa \operatorname{sech} \kappa, \kappa=-\frac{\sqrt{6 r s}}{6 \sqrt{p+q}} i\left(x_{1}+x_{2}-\frac{5 \sqrt{6 r s(p+q)}}{6}\right) i t \\
& u_{15}\left(x_{1}, x_{2}, t\right)=-\frac{s}{2} \tanh \kappa-\frac{s}{2} i \operatorname{sech} \kappa+\frac{s}{2} \tanh ^{2} \kappa+\frac{s}{2} i \tanh \kappa \operatorname{sech} \kappa, \kappa=\frac{\sqrt{6 r s}}{6 \sqrt{p+q}} i\left(x_{1}+x_{2}+\frac{5 \sqrt{6 r s(p+q)}}{6}\right) i t \\
& u_{16}\left(x_{1}, x_{2}, t\right)=-\frac{s}{2} \tanh \kappa-\frac{s}{2} i \operatorname{sech} \kappa+\frac{s}{2} \tanh ^{2} \kappa+\frac{s}{2} i \tanh \kappa \operatorname{sech} \kappa, \kappa=-\frac{\sqrt{6 r s}}{6 \sqrt{p+q}} i\left(x_{1}+x_{2}-\frac{5 \sqrt{6 r s(p+q)}}{6}\right) i t \\
& u_{17}\left(x_{1}, x_{2}, t\right)=\frac{s}{4}+\frac{s}{2} \tanh \kappa+\frac{s}{4} \tanh ^{2} \kappa, \kappa=\frac{\sqrt{6 r s}}{12 \sqrt{p+q}} i\left(x_{1}+x_{2}-\frac{5 \sqrt{6 r s(p+q)}}{6}\right) i t \\
& u_{18}\left(x_{1}, x_{2}, t\right)=\frac{s}{4}+\frac{s}{2} \tanh \kappa+\frac{s}{4} \tanh ^{2} \kappa, \kappa=-\frac{\sqrt{6 r s}}{12 \sqrt{p+q}} i\left(x_{1}+x_{2}+\frac{5 \sqrt{6 r s(p+q)}}{6}\right) i t \\
& u_{19}\left(x_{1}, x_{2}, t\right)=\frac{s}{4}-\frac{s}{2} \tanh \kappa+\frac{s}{4} \tanh ^{2} \kappa, \kappa=\frac{\sqrt{6 r s}}{12 \sqrt{p+q}} i\left(x_{1}+x_{2}+\frac{5 \sqrt{6 r s(p+q)}}{6}\right) i t \\
& u_{20}\left(x_{1}, x_{2}, t\right)=\frac{s}{4}+\frac{s}{2} \tanh \kappa+\frac{s}{4} \tanh ^{2} \kappa, \kappa=-\frac{\sqrt{6 r s}}{12 \sqrt{p+q}} i\left(x_{1}+x_{2}-\frac{5 \sqrt{6 r s(p+q)}}{6}\right) i t \\
& u_{21}\left(x_{1}, x_{2}, t\right)=\frac{3 s}{4}+\frac{s}{2} \tanh \kappa-\frac{s}{4} \tanh ^{2} \kappa, \kappa=\frac{\sqrt{6 r s}}{12 \sqrt{p+q}} i\left(x_{1}+x_{2}+\frac{5 \sqrt{6 r s(p+q)}}{6}\right) i t \\
& u_{22}\left(x_{1}, x_{2}, t\right)=\frac{3 s}{4}+\frac{s}{2} \tanh \kappa-\frac{s}{4} \tanh ^{2} \kappa, \kappa=-\frac{\sqrt{6 r s}}{12 \sqrt{p+q}} i\left(x_{1}+x_{2}-\frac{5 \sqrt{6 r s(p+q)}}{6}\right) i t \\
& u_{23}\left(x_{1}, x_{2}, t\right)=\frac{3 s}{4}-\frac{s}{2} \tanh \kappa-\frac{s}{4} \tanh ^{2} \kappa, \kappa=\frac{\sqrt{6 r s}}{12 \sqrt{p+q}} i\left(x_{1}+x_{2}-\frac{5 \sqrt{6 r s(p+q)}}{6}\right) i t \\
& u_{24}\left(x_{1}, x_{2}, t\right)=\frac{3 s}{4}-\frac{s}{2} \tanh \kappa-\frac{s}{4} \tanh ^{2} \kappa, \kappa=-\frac{\sqrt{6 r s}}{12 \sqrt{p 7 q}} i\left(x_{1}+x_{2}+\frac{5 \sqrt{6 r s(p+q)}}{6}\right) i t
\end{aligned}
$$


All determined solutions to the two dimensional Fisher equation are of the forms of various powers of multiplications of sech (.) and tanh (.) functions. Moreover, most of the solutions are of complex-valued traveling wave forms that change their position as time proceeds. One should note that some of the solutions given hyperbolic function forms can be represented in trigonometric forms by choosing compatible parameters.

In order to illustrate the complex-valued solutions, we chose a particular solution

$$
\begin{aligned}
u\left(x_{1}, x_{2}, t\right) & =1 / 5-i / 10 \sec \left(1 / 30 \sqrt{6} \sqrt{5}\left(x_{1}+x_{2}+\frac{i}{60} \sqrt{6} \sqrt{5} t\right)\right) \\
& +i / 10 \tan \left(1 / 30 \sqrt{6} \sqrt{5}\left(x_{1}+x_{2}+\frac{i}{60} \sqrt{6} \sqrt{5} t\right)\right) \\
& -1 / 10 \tan \left(1 / 30 \sqrt{6} \sqrt{5}\left(x_{1}+x_{2}+\frac{i}{60} \sqrt{6} \sqrt{5} t\right)\right) \sec \left(1 / 30 \sqrt{6} \sqrt{5}\left(x_{1}+x_{2}+\frac{i}{60} \sqrt{6} \sqrt{5} t\right)\right) \\
& +1 / 10\left(\tan \left(1 / 30 \sqrt{6} \sqrt{5}\left(x_{1}+x_{2}+\frac{i}{60} \sqrt{6} \sqrt{5} t\right)\right)\right)^{2}
\end{aligned}
$$

by using the parameter set $q=-1 / 10, r=-1 / 10, p=1 / 5, s=1 / 5$ in $u_{2}\left(x_{1}, x_{2}, t\right)$. The projections of real and imaginary components of the solution on the $\left(x[1], t, u\left(x_{1}, 0, t\right)\right)$ space are depicted in Fig 1(a) and Fig 1(b), respectively. The plots are generated in the finite domain $x_{1} \in[-100,100]$ in the time interval $0 \leq t \leq 1$.

A couple of initial solitary waves with different heights moves along $x_{1}$-axis as time proceeds in the real component of the solution, Fig 1(a). The highest one is observed to fade out as time goes. On the other hand, the highest solitary wave in the imaginary component gets higher as time proceeds, Fig 1(b).

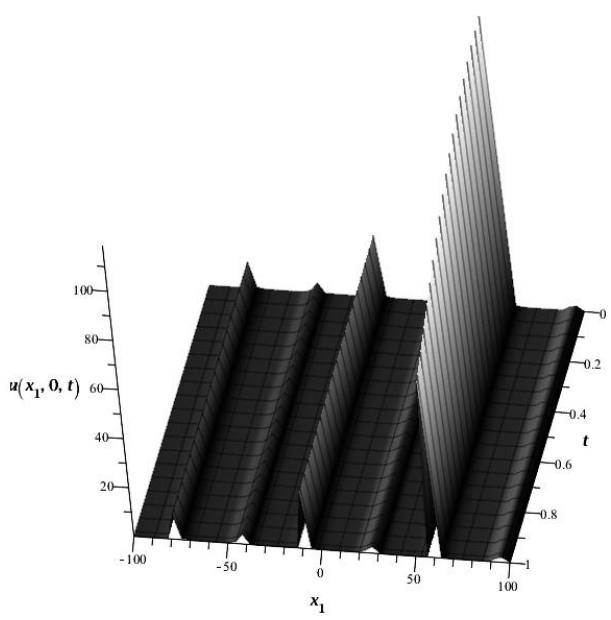

(a) real component

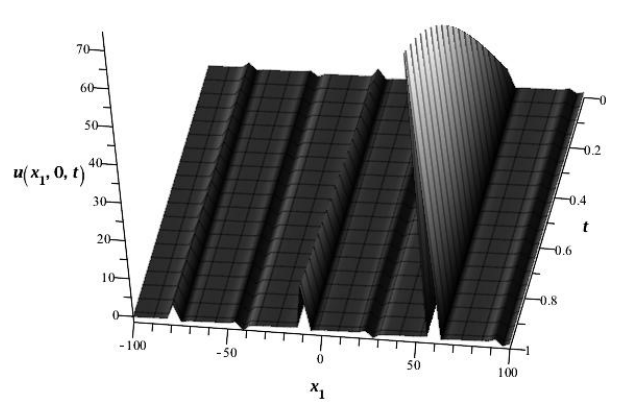

(b) imaginary component

Figure 1: The real and imaginary components of the particular solution $u_{2}\left(x_{1}, 0, t\right)$ for $\{q=-1 / 10, r=-1 / 10, p=1 / 5, s=1 / 5\}$ 


\section{Solutions to the Nagumo Equation}

The one dimensional form of the traveling wave transform

$$
u(z, t) \rightarrow U(\xi), \xi=a(z-\nu t),\left(z \text { is used instead of } x_{1} \text { only for simplicity }\right)
$$

reduces the Nagumo equation (3) to

$$
-a \nu U^{\prime}+p a^{2} U^{\prime \prime}+q U(1-U)(U-r)=0
$$

where' denotes derivative wrt $\xi$ in classical meaning. Since the balance procedure between $U^{\prime \prime}$ and $U^{3}$ results $Q=1$, the predicted solution to the Nagumo equation (3) is constructed in the form:

$$
U(w(\xi))=A_{0}+A_{1} \cos (w(\xi))+B_{1} \sin (w(\xi))
$$

Substitution of the predicted solution (23) into (22) and some simplifications using basic trigonometric identities is followed by rearranging the coefficients of trigonometric expressions. Equating each coefficient to zero leads

$$
\begin{aligned}
q r A_{0}{ }^{2}+q r B_{1}{ }^{2}-q A_{0}{ }^{3}-3 q A_{0} B_{1}{ }^{2}+a \nu A_{1}-q r A_{0}+q A_{0}{ }^{2}+q B_{1}{ }^{2} & =0 \\
-p a^{2} B_{1}+2 q r A_{0} B_{1}-3 q A_{0}{ }^{2} B_{1}-q B_{1}{ }^{3}-q r B_{1}+2 q A_{0} B_{1} & =0 \\
p a^{2} B_{1}-3 q A_{1}{ }^{2} B_{1}+q B_{1}{ }^{3} & =0 \\
2 q r A_{1} B_{1}-6 q A_{0} A_{1} B_{1}-a \nu B_{1}+2 q A_{1} B_{1} & =0 \\
2 p a^{2} A_{1}-q A_{1}{ }^{3}+3 q B_{1}{ }^{2} A_{1} & =0 \\
q A_{1}{ }^{2} r-q r B_{1}{ }^{2}-3 q A_{1}{ }^{2} A_{0}+3 q A_{0} B_{1}{ }^{2}-a \nu A_{1}+q A_{1}{ }^{2}-q B_{1}{ }^{2} & =0 \\
-2 p a^{2} A_{1}+2 q r A_{0} A_{1}-3 q A_{0}{ }^{2} A_{1}-3 q B_{1}{ }^{2} A_{1}-q r A_{1}+2 q A_{0} A_{1} & =0
\end{aligned}
$$

The solution of this algebraic system for $a \neq 0, \nu \neq 0, A_{0}, A_{1}$ and $B_{1}$ at least one of $A_{1}$ and $B_{1}$ are non zero tabulated in Table 2. The solutions to the Nagumo equation are constructed in finite series form of tanh (.) and sech (.) functions. 
Table 2: The solution of the algebraic system of equations derived for Nagumo equation

\begin{tabular}{|c|c|c|c|c|}
\hline$a$ & $\nu$ & $A_{0}$ & $A_{1}$ & $B_{1}$ \\
\hline$\frac{(r-1) \sqrt{q}}{\sqrt{2 p}}$ & $\frac{(r+1) \sqrt{p q}}{\sqrt{2}}$ & $\frac{r+1}{2}$ & $\frac{1-r}{2}$ & $\frac{r-1}{2} i$ \\
\hline$-\left(\begin{array}{l}(r-1) \sqrt{q} \\
\end{array}\right.$ & $-\left(\begin{array}{c}(r+1) \sqrt{p q} \\
\end{array}\right.$ & $r+1$ & $\underline{1-r}$ & $\underline{r-1} i$ \\
\hline$\frac{\sqrt{2 p}}{(r-1) \sqrt{q}}$ & $(r+1) \sqrt{\frac{1}{p q}}$ & $\begin{array}{c}\overline{2} \\
r+1\end{array}$ & $\begin{array}{c}\overline{2} \\
1-r\end{array}$ & $\begin{array}{l}\overline{2}_{r-1} \\
{ }^{2} \text {. }\end{array}$ \\
\hline$\sqrt{\sqrt{2 p}}$ & & $\frac{1+1}{2}$ & $\frac{1}{2}$ & $-\frac{r-1}{2} i$ \\
\hline$\frac{(r-1) \sqrt{q}}{\sqrt{2 p}}$ & $-\frac{(r+1) \sqrt{p q}}{\sqrt{2}}$ & $\frac{r+1}{2}$ & $\frac{1-r}{2}$ & $-\frac{r-1}{2} i$ \\
\hline$\frac{(r-1) \sqrt{q}}{\sqrt{2 n}}$ & $-\frac{(r+1) \sqrt{p q}}{\sqrt{2}}$ & $\frac{r+1}{2}$ & $\frac{1-r}{2}$ & $\frac{r-1}{2} i$ \\
\hline$-\frac{(r-1) \sqrt{q}}{\sqrt{2}}$ & $\frac{(r+1) \sqrt{p q}}{\underline{\sqrt{n}}}$ & $\frac{r+1}{2}$ & $-\frac{1-r}{2}$ & $\frac{r-1}{2} i$ \\
\hline$(r-1) \sqrt{q}$ & $-\begin{array}{c}(r+1) \sqrt{p q} \\
-\end{array}$ & $r+1$ & $\underline{1-r}$ & $-\frac{r-1}{-} i$ \\
\hline $\begin{array}{r}\sqrt{2 p} \\
(r-1) \sqrt{q} \\
\end{array}$ & $\begin{array}{c}\sqrt{2} \\
(r+1) \sqrt{p q} \\
\end{array}$ & $\begin{array}{c}2 \\
r+1 \\
\end{array}$ & $\begin{array}{l}2 \\
1-r\end{array}$ & $\begin{array}{c}2 \\
r-1\end{array}$ \\
\hline $\begin{array}{c}\sqrt{2 p} \\
r \sqrt{q}\end{array}$ & $\begin{array}{r}\sqrt{2} \\
(r-2) \sqrt{p q}\end{array}$ & $\overline{2}$ & $-\frac{\overline{2}}{r}$ & $-\frac{2}{2}^{l}$ \\
\hline$\frac{\sqrt{4}}{\sqrt{2 p}}$ & $\sqrt{2}$ & $\frac{r}{2}$ & $-\frac{r}{2}$ & $\frac{r}{2} i$ \\
\hline$-\frac{r \sqrt{ } q}{\sqrt{2 p}}$ & $-\frac{(r-2) \sqrt{p q}}{\sqrt{2}}$ & $\frac{r}{2}$ & $-\frac{r}{2}$ & $\frac{r}{2} i$ \\
\hline$\frac{r \sqrt{q}}{\sqrt{2 p}}$ & $\frac{(r-2) \sqrt{p q}}{\sqrt{2}}$ & $\frac{r}{2}$ & $-\frac{r}{2}$ & $-\frac{r}{2} i$ \\
\hline$-\frac{r \sqrt{q}}{\sqrt{2 p}}$ & $-\frac{(r-2) \sqrt{p q}}{\sqrt{2}}$ & $\frac{r}{2}$ & $-\frac{r}{2}$ & $-\frac{r}{2} i$ \\
\hline$\frac{r \sqrt{q}}{\sqrt{2 p}}$ & $-\frac{(r-2) \sqrt{p q}}{\sqrt{2}}$ & $\frac{r}{2}$ & $\frac{r}{2}$ & $\frac{r}{2} i$ \\
\hline$-\frac{r \sqrt{q}}{\sqrt{2 n}}$ & $\frac{(r-2) \sqrt{p q}}{\sqrt{2}}$ & $\frac{r}{2}$ & $\frac{r}{2}$ & $\frac{r}{2} i$ \\
\hline$r \sqrt{q}$ & $-(r-2) \sqrt{p q}$ & $\underline{T}$ & $\frac{r}{a}$ & $-\frac{r}{-r} i$ \\
\hline $\begin{array}{l}\sqrt{2 p} \\
r \sqrt{q}\end{array}$ & $\begin{array}{l}\sqrt{2} \\
(r-2) \sqrt{p q}\end{array}$ & 2 & 2 & $\begin{array}{l}2 \\
r \\
r\end{array}$ \\
\hline$-\frac{\sqrt{2 p}}{\sqrt{2 p}}$ & $\begin{array}{r}\sqrt{2} \\
r-1)\end{array}$ & $\overline{2}$ & $\overline{2}$ & $-\frac{1}{2} l$ \\
\hline$\frac{\sqrt{q}}{\sqrt{2 p}}$ & $\frac{(2 r-1) \sqrt{p q}}{\sqrt{2}}$ & $\frac{1}{2}$ & $\frac{1}{2}$ & $\frac{1}{2} i$ \\
\hline$-\frac{\sqrt{q}}{\sqrt{2 p}}$ & $-\frac{(2 r-1) \sqrt{p q}}{\sqrt{2}}$ & $\frac{1}{2}$ & $\frac{1}{2}$ & $\frac{1}{2} i$ \\
\hline$\frac{\sqrt{q}}{\sqrt{2 p}}$ & $\frac{(2 r-1) \sqrt{p q}}{\sqrt{2}}$ & $\frac{1}{2}$ & $\frac{1}{2}$ & $-\frac{1}{2} i$ \\
\hline$\sqrt{q}$ & $-(2 r-1) \sqrt{p q}$ & 1 & 1 & \\
\hline$-\frac{\sqrt{2 p}}{\sqrt{q}}$ & $-\frac{\sqrt{2}}{(2 r-1) \sqrt{p q}}$ & $\overline{2}$ & $\overline{2}$ & $-\overline{2} l$ \\
\hline$\frac{\sqrt{q}}{\sqrt{2 p}}$ & $-\frac{(2 T-1) \sqrt{ } p q}{\sqrt{2}}$ & $\frac{1}{2}$ & $-\frac{1}{2}$ & $\frac{1}{2} i$ \\
\hline$-\frac{\sqrt{q}}{\sqrt{2 p}}$ & $\frac{(2 r-1) \sqrt{p q}}{\sqrt{2}}$ & $\frac{1}{2}$ & $-\frac{1}{2}$ & $\frac{1}{2} i$ \\
\hline$\frac{\sqrt{q}}{\sqrt{2 n}}$ & $-\frac{(2 r-1) \sqrt{p q}}{\sqrt{2}}$ & $\frac{1}{2}$ & $-\frac{1}{2}$ & $-\frac{1}{2} i$ \\
\hline$-\frac{\sqrt{q}}{\sqrt{2 p}}$ & $\frac{(2 r-1) \sqrt{p q}}{\sqrt{2}}$ & $\frac{1}{2}$ & $-\frac{1}{2}$ & $-\frac{1}{2} i$ \\
\hline$\underline{(r-1) \sqrt{2 q}}$ & $\frac{(r+1) \sqrt{p q}}{\sqrt{5}}$ & $\frac{1+r}{2}$ & $\frac{1-r}{2}$ & 0 \\
\hline $\begin{array}{c}4 \sqrt{p} \\
(r-1) \sqrt{2 q}\end{array}$ & $\begin{array}{c}\sqrt{2} \\
(r+1) \sqrt{p q}\end{array}$ & $1+r$ & $1-r$ & 0 \\
\hline $4 \sqrt{p}$ & $\sqrt{2}$ & $\overline{2}$ & $\overline{2}$ & 0 \\
\hline$\frac{(r-1) \sqrt{2 q}}{4 \sqrt{p}}$ & $-\frac{(r+1) \sqrt{p q}}{\sqrt{2}}$ & $\frac{1+r}{2}$ & $-\frac{1-r}{2}$ & 0 \\
\hline$-\underline{(r-1) \sqrt{2 q}}$ & $\frac{(r+1) \sqrt{p q}}{}$ & $\underline{1+r}$ & $-\frac{1-r}{2}$ & 0 \\
\hline $\begin{array}{r}4 \sqrt{p} \\
r \sqrt{2 q}\end{array}$ & $\begin{array}{c}\sqrt{2} \\
(r-2) \sqrt{p q}\end{array}$ & 2 & 2 & \\
\hline$\overline{4 \sqrt{p}}$ & $\sqrt{2}$ & $\frac{1}{2}$ & $-\frac{1}{2}$ & 0 \\
\hline$-\frac{r \sqrt{2 q}}{4 \sqrt{p}}$ & $-\frac{(r-2) \sqrt{p q}}{\sqrt{2}}$ & $\frac{r}{2}$ & $-\frac{r}{2}$ & 0 \\
\hline$\frac{r \sqrt{2 q}}{4 \sqrt{p}}$ & $-\frac{(r-2) \sqrt{p q} 10}{\sqrt{2}}$ & $\frac{r}{r}$ & $\frac{r}{2}$ & 0 \\
\hline $\begin{array}{r}4 \sqrt{p} \\
-r \sqrt{2 q} \\
\end{array}$ & $\begin{array}{c}\sqrt{2} \\
(r-2) \sqrt{p q} \\
\end{array}$ & & $\frac{1}{-}$ & 0 \\
\hline$\frac{4 \sqrt{p}}{\sqrt{2 q}}$ & & $\overline{2}$ & $\overline{2}$ & U \\
\hline$\frac{v \angle q}{4 \sqrt{p}}$ & $\frac{(2 r-1) \sqrt{ } p q}{\sqrt{2}}$ & $\frac{1}{2}$ & $\frac{1}{2}$ & 0 \\
\hline$-\frac{\sqrt{2 q}}{4 \sqrt{p}}$ & $-\frac{(2 r-1) \sqrt{p q}}{\sqrt{2}}$ & $\frac{1}{2}$ & $\frac{1}{2}$ & 0 \\
\hline$\frac{\sqrt{2 q}}{4 \sqrt{n}}$ & $(2 r-1) \sqrt{p q}$ & $\frac{1}{2}$ & $-\frac{1}{2}$ & 0 \\
\hline $\begin{array}{r}4 \sqrt{p} \\
-\quad \sqrt{2 q} \\
\end{array}$ & $\begin{array}{c}\sqrt{ } 2 \\
(2 r-1) \sqrt{p q} \\
\end{array}$ & $\underline{1}$ & $-\frac{1}{3}$ & 0 \\
\hline $4 \sqrt{p}$ & $\sqrt{2}$ & 2 & 2 & \\
\hline
\end{tabular}


These solutions are expressed explicitly as

$$
\begin{aligned}
& u_{1}(z, t)=\frac{r+1}{2}-\frac{1-r}{2} \tanh \kappa+\frac{r-1}{2} i \operatorname{sech} \kappa, \kappa=\frac{(r-1) \sqrt{q}}{\sqrt{2 p}}\left(z-\frac{(r+1) \sqrt{p q}}{\sqrt{2}} t\right) \\
& u_{2}(z, t)=\frac{r+1}{2}-\frac{1-r}{2} \tanh \kappa+\frac{r-1}{2} i \operatorname{sech} \kappa, \kappa=-\frac{(r-1) \sqrt{q}}{\sqrt{2 p}}\left(z+\frac{(r+1) \sqrt{p q}}{\sqrt{2}} t\right) \\
& u_{3}(z, t)=\frac{r+1}{2}-\frac{1-r}{2} \tanh \kappa-\frac{r-1}{2} i \operatorname{sech} \kappa, \kappa=\frac{(r-1) \sqrt{q}}{\sqrt{2 p}}\left(z-\frac{(r+1) \sqrt{p q}}{\sqrt{2}} t\right) \\
& u_{4}(z, t)=\frac{r+1}{2}-\frac{1-r}{2} \tanh \kappa-\frac{r-1}{2} i \operatorname{sech} \kappa, \kappa=-\frac{(r-1) \sqrt{q}}{\sqrt{2 p}}\left(z+\frac{(r+1) \sqrt{p q}}{\sqrt{2}} t\right) \\
& u_{5}(z, t)=\frac{r+1}{2}-\frac{1-r}{2} \tanh \kappa-\frac{r-1}{2} i \operatorname{sech} \kappa, \kappa=\frac{(r-1) \sqrt{q}}{\sqrt{2 p}}\left(z+\frac{(r+1) \sqrt{p q}}{\sqrt{2}} t\right) \\
& u_{6}(z, t)=\frac{r+1}{2}+\frac{1-r}{2} \tanh \kappa+\frac{r-1}{2} i \operatorname{sech} \kappa, \kappa=-\frac{(r-1) \sqrt{q}}{\sqrt{2 p}}\left(z-\frac{(r+1) \sqrt{p q}}{\sqrt{2}} t\right) \\
& u_{7}(z, t)=\frac{r+1}{2}-\frac{1-r}{2} \tanh \kappa-\frac{r-1}{2} i \operatorname{sech} \kappa, \kappa=\frac{(r-1) \sqrt{q}}{\sqrt{2 p}}\left(z+\frac{(r+1) \sqrt{p q}}{\sqrt{2}} t\right) \\
& u_{8}(z, t)=\frac{r+1}{2}+\frac{1-r}{2} \tanh \kappa-\frac{r-1}{2} i \operatorname{sech} \kappa, \kappa=-\frac{(r-1) \sqrt{q}}{\sqrt{2 p}}\left(z-\frac{(r+1) \sqrt{p q}}{\sqrt{2}} t\right) \\
& u_{9}(z, t)=\frac{r}{2}+\frac{-r}{2} \tanh \kappa+\frac{r}{2} i \operatorname{sech} \kappa, \kappa=\frac{(r) \sqrt{q}}{\sqrt{2 p}}\left(z-\frac{(r-2) \sqrt{p q}}{\sqrt{2}} t\right) \\
& u_{10}(z, t)=\frac{r}{2}+\frac{-r}{2} \tanh \kappa+\frac{r}{2} i \operatorname{sech} \kappa, \kappa=-\frac{(r) \sqrt{q}}{\sqrt{2 p}}\left(z+\frac{(r-2) \sqrt{p q}}{\sqrt{2}} t\right) \\
& u_{11}(z, t)=\frac{r}{2}+\frac{-r}{2} \tanh \kappa-\frac{r}{2} i \operatorname{sech} \kappa, \kappa=\frac{(r) \sqrt{q}}{\sqrt{2 p}}\left(z-\frac{(r-2) \sqrt{p q}}{\sqrt{2}} t\right) \\
& u_{12}(z, t)=\frac{r}{2}+\frac{-r}{2} \tanh \kappa-\frac{r}{2} i \operatorname{sech} \kappa, \kappa=-\frac{(r) \sqrt{q}}{\sqrt{2 p}}\left(z+\frac{(r-2) \sqrt{p q}}{\sqrt{2}} t\right) \\
& u_{13}(z, t)=\frac{r}{2}-\frac{-r}{2} \tanh \kappa+\frac{r}{2} i \operatorname{sech} \kappa, \kappa=\frac{(r) \sqrt{q}}{\sqrt{2 p}}\left(z+\frac{(r-2) \sqrt{p q}}{\sqrt{2}} t\right) \\
& u_{14}(z, t)=\frac{r}{2}-\frac{-r}{2} \tanh \kappa+\frac{r}{2} i \operatorname{sech} \kappa, \kappa=-\frac{(r) \sqrt{q}}{\sqrt{2 p}}\left(z-\frac{(r-2) \sqrt{p q}}{\sqrt{2}} t\right) \\
& u_{15}(z, t)=\frac{r}{2}-\frac{-r}{2} \tanh \kappa-\frac{r}{2} i \operatorname{sech} \kappa, \kappa=\frac{(r) \sqrt{q}}{\sqrt{2 p}}\left(z+\frac{(r-2) \sqrt{p q}}{\sqrt{2}} t\right) \\
& u_{16}(z, t)=\frac{r}{2}-\frac{-r}{2} \tanh \kappa-\frac{r}{2} i \operatorname{sech} \kappa, \kappa=-\frac{(r) \sqrt{q}}{\sqrt{2 p}}\left(z-\frac{(r-2) \sqrt{p q}}{\sqrt{2}} t\right) \\
& u_{17}(z, t)=\frac{1}{2}-\frac{1}{2} \tanh \kappa+\frac{1}{2} i \operatorname{sech} \kappa, \kappa=\frac{\sqrt{q}}{\sqrt{2 p}}\left(z-\frac{(2 r-1) \sqrt{p q}}{\sqrt{2}} t\right) \\
& u_{18}(z, t)=\frac{1}{2}-\frac{1}{2} \tanh \kappa+\frac{1}{2} i \operatorname{sech} \kappa, \kappa=-\frac{\sqrt{q}}{\sqrt{2 p}}\left(z+\frac{(2 r-1) \sqrt{p q}}{\sqrt{2}} t\right) \\
& u_{19}(z, t)=\frac{1}{2}-\frac{1}{2} \tanh \kappa-\frac{1}{2} i \operatorname{sech} \kappa, \kappa=\frac{\sqrt{q}}{\sqrt{2 p}}\left(z-\frac{(2 r-1) \sqrt{p q}}{\sqrt{2}} t\right) \\
& u_{20}(z, t)=\frac{1}{2}-\frac{1}{2} \tanh \kappa-\frac{1}{2} i \operatorname{sech} \kappa, \kappa=-\frac{\sqrt{q}}{\sqrt{2 p}}\left(z+\frac{(2 r-1) \sqrt{p q}}{\sqrt{2}} t\right) \\
& u_{21}(z, t)=\frac{1}{2}+\frac{1}{2} \tanh \kappa+\frac{1}{2} i \operatorname{sech} \kappa, \kappa=\frac{\sqrt{q}}{\sqrt{2 p}}\left(z+\frac{(2 r-1) \sqrt{p q}}{\sqrt{2}} t\right) \\
& u_{22}(z, t)=\frac{1}{2}+\frac{1}{2} \tanh \kappa+\frac{1}{2} i \operatorname{sech} \kappa, \kappa=-\frac{\sqrt{q}}{\sqrt{2 p}}\left(z-\frac{(2 r-1) \sqrt{p q}}{\sqrt{2}} t\right) \\
& u_{23}(z, t)=\frac{1}{2}+\frac{1}{2} \tanh \kappa-\frac{1}{2} i \operatorname{sech} \kappa, \kappa=\frac{\sqrt{q}}{\sqrt{2 p}}\left(z+\frac{(2 r-1) \sqrt{p q}}{\sqrt{2}} t\right) \\
& u_{24}(z, t)=\frac{1}{2}+\frac{1}{2} \tanh \kappa-\frac{1}{2} i \operatorname{sech} \kappa, \kappa=-\frac{\sqrt{q}}{\sqrt{2 p}}\left(z-\frac{(2 r-1) \sqrt{p q}}{\sqrt{2}} t\right) \\
& u_{25}(z, t)=\frac{1+r}{2}-\frac{1-r}{2} \tanh \kappa, \kappa=\frac{(r-1) \sqrt{2 q}}{4 \sqrt{p}}\left(z-\frac{(r+1) \sqrt{p q}}{\sqrt{2}} t\right) \\
& u_{26}(z, t)=\frac{1+r}{2}-\frac{1-r}{2} \tanh \kappa, \kappa=-\frac{(1-1) \sqrt{2 q}}{4 \sqrt{p}}\left(z+\frac{(r+1) \sqrt{p q}}{\sqrt{2}} t\right) \\
& u_{27}(z, t)=\frac{1+r}{2}+\frac{1-r}{2} \tanh \kappa, \kappa=\frac{(r-1) \sqrt{2 q}}{4 \sqrt{p}}\left(z+\frac{(r+1) \sqrt{p q}}{\sqrt{2}} t\right) \\
& u_{28}(z, t)=\frac{1+r}{2}+\frac{1-r}{2} \tanh \kappa, \kappa=-\frac{(r-1) \sqrt{2 q}}{4 \sqrt{p}}\left(z-\frac{(r+1) \sqrt{p q}}{\sqrt{2}} t\right)
\end{aligned}
$$




$$
\begin{aligned}
& u_{29}(z, t)=\frac{r}{2}+\frac{r}{2} \tanh \kappa, \kappa=\frac{(r) \sqrt{2 q}}{4 \sqrt{p}}\left(z-\frac{(r-2) \sqrt{p q}}{\sqrt{2}} t\right) \\
& u_{30}(z, t)=\frac{r}{2}+\frac{r}{2} \tanh \kappa, \kappa=-\frac{(r) \sqrt{2 q}}{4 \sqrt{p}}\left(z+\frac{(r-2) \sqrt{p q}}{\sqrt{2}} t\right) \\
& u_{31}(z, t)=\frac{r}{2}-\frac{r}{2} \tanh \kappa, \kappa=\frac{(r) \sqrt{2 q}}{4 \sqrt{p}}\left(z-\frac{(r-2) \sqrt{p q}}{\sqrt{2}} t\right) \\
& u_{32}(z, t)=\frac{r}{2}-\frac{r}{2} \tanh \kappa, \kappa=-\frac{(r) \sqrt{2 q}}{4 \sqrt{p}}\left(z+\frac{(r-2) \sqrt{p q}}{\sqrt{2}} t\right) \\
& u_{33}(z, t)=\frac{1}{2}-\frac{1}{2} \tanh \kappa, \kappa=\frac{\sqrt{2 q}}{4 \sqrt{p}}\left(z-\frac{(2 r-1) \sqrt{p q}}{\sqrt{2}} t\right) \\
& u_{34}(z, t)=\frac{1}{2}-\frac{1}{2} \tanh \kappa, \kappa=-\frac{\sqrt{2 q}}{4 \sqrt{p}}\left(z+\frac{(2 r-1) \sqrt{p q}}{\sqrt{2}} t\right) \\
& u_{35}(z, t)=\frac{1}{2}+\frac{1}{2} \tanh \kappa, \kappa=\frac{\sqrt{2 q}}{4 \sqrt{p}}\left(z-\frac{(2 r-1) \sqrt{p q}}{\sqrt{2}} t\right) \\
& u_{36}(z, t)=\frac{1}{2}+\frac{1}{2} \tanh \kappa, \kappa=-\frac{\sqrt{2 q}}{4 \sqrt{p}}\left(z+\frac{(2 r-1) \sqrt{p q}}{\sqrt{2}} t\right)
\end{aligned}
$$

Real and imaginary components of the particular solution $u_{2}(z, t)$ for the parameters $q=1, r=-i / 2, p=-1$ are depicted in Fig 2(a)-2(b), respectively. The finite problem domain $z \in[0,25]$ is used during first 20 unit times, Fig $2(\mathrm{a})$. The real component of the solution is a positive pulse positioned between $0<z<5$ approximately. It gets larger for a while. Then, suddenly it becomes a negative pulse. It fades out after some time. It comes into existence at about $t=5$ with positive height positioned at about $z=11$. It shows the same behavior, and fades out again. The occurrence and fade out are repeated after $t=20$. A similar graphic is also observed in the imaginary component plot, Fig 2(b).

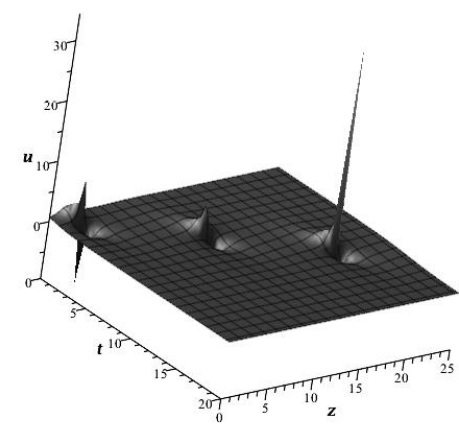

(a) real component

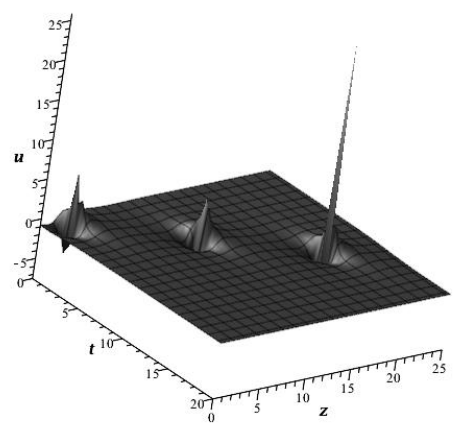

(b) imaginary component

Figure 2: The real and imaginary components of the particular solution $u_{2}(z, t)$ for $\{q=1, r=-i / 2, p=-1\}$ 


\section{Conclusion}

The extension method based the Sine-Gordon equation is extended to multi dimension. Compatible traveling wave transforms are used to reduce the governing equations to some ODEs. The predicted solutions solving the resultant ODEs are substituted into the equations to determine the relations among the parameters. The homogeneous balancing procedure has a significant role on the shape of the solutions.

The complex-valued solutions to two dimensional Fisher and one dimensional Nagumo equations are explicitly expressed in finite series form in terms of multiplications of some hyperbolic functions with different powers.

Some particular solutions of both equations are depicted to illustrate the motions. Both real and imaginary components are examined briefly in both cases. 


\section{References}

[1] Fisher, R. A., The advance of advantageous genes, Ann. of Eugenics, 7, 355369, 1937.

[2] Murray, J. D., Mathematical Biology: I. An Introduction, Springer, 2002.

[3] Gilding, B.H., Kersner R. (2001), Traveling Waves in Nonlinear Diffusionconvection-reaction, University of Twente, Memorandum No. 1585.

[4] Kolmogorov, A., Petrovsky, I., Piskounov, N., Etude de l'équation de la diffusion avec croissance de la quantité de matière et son application à un problème biologique, Bull Univ État Moscou Sér Int A, 1, 1-26, 1937.

[5] Ablowitz, M. J., \& Zeppetella, A. (1979). Explicit solutions of Fisher's equation for a special wave speed. Bulletin of Mathematical Biology, 41(6), 835840.

[6] Kudryashov, N. A. (2005). Exact solitary waves of the Fisher equation. Physics Letters A, 342(1), 99-106.

[7] Öziş, T., \& Köroğlu, C. (2008). A novel approach for solving the Fisher equation using Exp-function method. Physics Letters A, 372(21), 3836-3840.

[8] Feng, Z., \& Li, Y. (2006). Complex traveling wave solutions to the Fisher equation. Physica A: Statistical Mechanics and its Applications, 366, 115123.

[9] Kudryashov, N. A. (2012). One method for finding exact solutions of nonlinear differential equations. Communications in Nonlinear Science and Numerical Simulation, 17(6), 2248-2253.

[10] Aronson, D. G., \& Weinberger, H. F. (1978). Multidimensional nonlinear diffusion arising in population genetics. Advances in Mathematics, 30(1), 3376.

[11] Zhi-Xiong, C., \& Ben-Yu, G. (1992). Analytic solutions of the Nagumo equation. IMA journal of applied mathematics, 48(2), 107-115.

[12] Tauseef Mohyud-Din, S., Khan, Y., Faraz, N., \& Yıldırım, A. (2012). Expfunction method for solitary and periodic solutions of Fitzhugh-Nagumo equation. International Journal of Numerical Methods for Heat \& Fluid Flow, $22(3), 335-341$. 
[13] Dehghan, M., Manafian Heris, J., \& Saadatmandi, A. (2011). Application of the Exp-function method for solving a partial differential equation arising in biology and population genetics. International Journal of Numerical Methods for Heat \& Fluid Flow, 21(6), 736-753.

[14] Ma, W. X., \& Fuchssteiner, B. (1996). Explicit and exact solutions to a Kolmogorov-Petrovskii-Piskunov equation. International Journal of NonLinear Mechanics, 31(3), 329-338.

[15] Kawahara, T., \& Tanaka, M. (1983). Interactions of traveling fronts: an exact solution of a nonlinear diffusion equation. Physics Letters A, 97(8), 311-314.

[16] Jawad, A. J. A. M., Petkovich, M. D., \& Biswas, A. (2010). Modified simple equation method for nonlinear evolution equations. Applied Mathematics and Computation, 217(2), 869-877.

[17] Li, H., \& Guo, Y. (2006). New exact solutions to the Fitzhugh-Nagumo equation. Applied Mathematics and Computation, 180(2), 524-528.

[18] Yan, C. (1996), A simple transformation for nonlinear waves, Physics Letters A, 224, 77-84. 